\title{
Chondrogenic Differentiation of Mesenchymal Stem Cells on Glycosaminoglycan-Mimetic Peptide Nanofibers
}

\author{
Seher Ustun Yaylaci, Merve Sen, Ozlem Bulut, Elif Arslan, Mustafa O. Guler,* and Ayse B. Tekinay* \\ Institute of Materials Science and Nanotechnology, National Nanotechnology Research Center (UNAM), Bilkent University, Ankara \\ 06800, Turkey
}

\section{Supporting Information}

ABSTRACT: Glycosaminoglycans (GAGs) are important extracellular matrix components of cartilage tissue and provide biological signals to stem cells and chondrocytes for development and functional regeneration of cartilage. Among their many functions, particularly sulfated glycosaminoglycans bind to growth factors and enhance their functionality through enabling growth factor-receptor interactions. Growth factor binding ability of the native sulfated glycosaminoglycans can be incorporated into the synthetic scaffold matrix through functionalization with specific chemical moieties. In this study, we used peptide amphiphile nanofibers functionalized with the chemical groups of native glycosaminoglycan molecules such as sulfonate, carboxylate and hydroxyl to induce the chondrogenic differentiation of rat mesenchymal stem cells (MSCs). The MSCs cultured on GAG-mimetic peptide nanofibers formed cartilage-like nodules and deposited cartilage-specific matrix components by day 7, suggesting that the GAG-mimetic peptide nanofibers effectively facilitated their commitment into the chondrogenic lineage. Interestingly, the chondrogenic differentiation degree was manipulated with the sulfonation degree of the nanofiber system. The GAG-mimetic peptide nanofibers network presented here serve as a tailorable bioactive and bioinductive platform for stem-cell-based cartilage regeneration studies.

KEYWORDS: chondrogenic differentiation, in vitro condensation, mesenchymal stem cells, peptide amphiphile nanofiber, GAG-mimetic

\section{INTRODUCTION}

Mesenchymal stem cells (MSCs) are commonly used for cellbased regenerative therapies because of their availability, ease of culture, and capacity for self-renewal and multilineage differentiation. ${ }^{1,2}$ Commitment and maturation of stem cells are strictly regulated through soluble and physical factors found in the tissue microenvironment, which should be provided to facilitate their in vitro differentiation into specific lineages. As such, various culture medium components are used to direct the lineage commitment of stem cells in natural and synthetic scaffolds. $^{3-6}$ In addition to soluble factors, cellular differentiation can also be altered by the chemical composition and biomechanical properties of the extracellular environment. Tissues such as bone and cartilage are especially reliant on a specific set of biochemical and mechanical cues for their repair; consequently, scaffolds for cartilage regeneration must present an appropriate combination of physical characteristics and soluble factors to produce an ideal environment for chondrogenic differentiation.

MSCs have been reported to undergo in vitro chondrogenesis and deposit cartilage-specific matrix molecules in a variety of natural and synthetic materials, especially when cultured in the presence of a precise set of growth factors. Growth factors can be provided to the culture environment through several means: they may, for example, be physically encapsulated within the matrix, added into the culture medium, released over time by growth factor release vectors, or covalently attached to the scaffold in random or specific orientations. $^{8-11}$ However, growth factors are expensive and sensitive, and often lose their bioactivity during sterilization procedures. In addition, they have a narrow $\mathrm{pH}$ tolerance and are susceptible to proteolytic degradation, which leads to their rapid clearance under in vivo conditions. ${ }^{12}$ The clinical use of growth factors is also a contentious issue, as some are known to be proto-oncogenic. ${ }^{13,14} \mathrm{New}$ generation biomaterials that can use the endogenously produced growth factors to facilitate chondrogenic differentiation could therefore enhance the efficiency and clinical potential of regenerative scaffolds. ${ }^{15,16}$

The bioactivity of growth factors is regulated through their interactions with extracellular matrix elements. In particular, sulfated glycosaminoglycans such as heparan sulfate are capable of facilitating the immobilization and release of growth factors through their negatively charged sulfate and carboxylate groups. A number of recent studies have demonstrated that growth

Received: February 18, 2016

Accepted: March 23, 2016

Published: March 23, 2016 


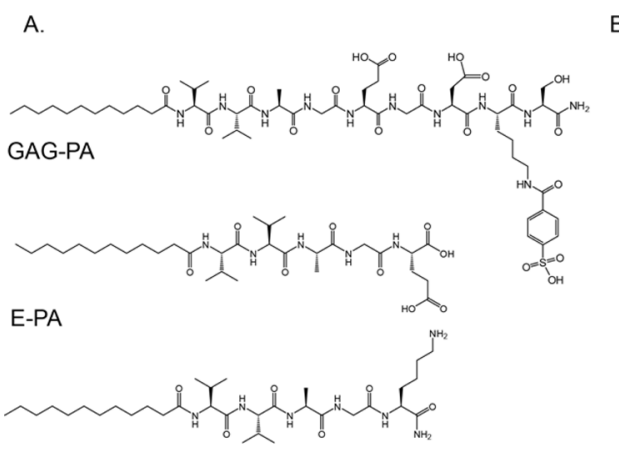

K-PA
B.

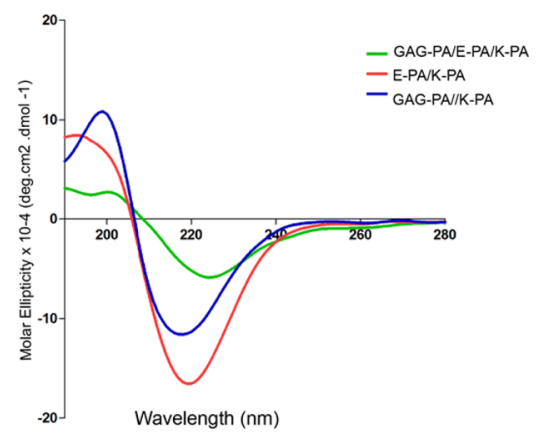

C.
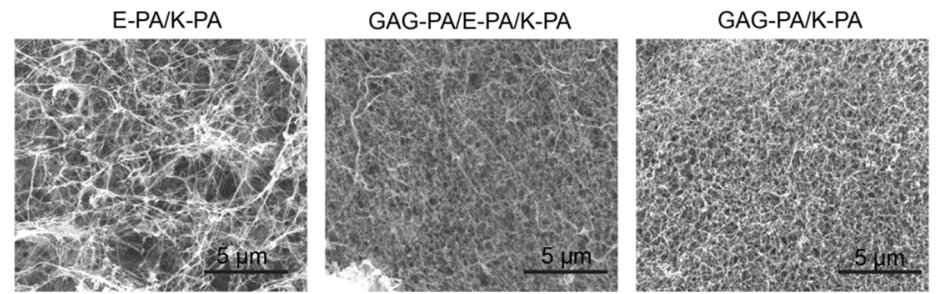

Figure 1. Self-assembly of peptide amphiphile molecules into nanofibrous networks. (A) Chemical presentation of peptide amphiphile molecules. Lauryl-VVAGEGDKS-Am (GAG-PA), Lauryl-VVAGE (E-PA), and Lauryl-VVAGK-Am (K-PA). (B) Circular dichroism spectra of the nanofibers showing $\beta$-sheet-like structure. (C) SEM images showing extracellular matrix mimetic morphology of nanofiber networks.

factor sequestration can also be performed by biomaterial scaffolds that incorporate glycosaminoglycans in their structure, allowing these materials to modulate the biological responses of cells. ${ }^{17-20}$ The addition of heparan sulfate, heparin, or dermatan sulfate enhanced the formation of chondrogenic cell aggregates and the sulfate-bearing domain of perlecan was responsible for the in vitro aggregation and chondrogenic activation of $\mathrm{C} 3 \mathrm{H} 10 \mathrm{~T} 1 / 2$ cells. ${ }^{21}$ However, the use of heterogeneous combinations of glycosaminoglycans in unknown ratios prevents the in-depth analysis of structure-function relationships and complicates the clinical use of these materials because of concerns involving off-target effects. ${ }^{22,23}$ In addition, glycosaminoglycans are often covalently cross-linked to hydrogels, which introduces toxic side products into the material matrix, restricts the conformation of the scaffoldbound biomolecules, and weakens the overall biological functionality of the system. A "reductionist glycosaminoglycan-mimetic approach" involving the use of small chemical groups has been proposed as an alternative to the cross-linking of glycosaminoglycans and was shown to be effective: sulfate/ sulfonate groups, for example, can affect cytoskeletal organization and motility of mesenchymal stem cells in a short period of culture ${ }^{24}$ and can induce the chondrogenic differentiation of stem cells into chondroprogenitors under in vitro conditions. ${ }^{25}$

Here, we report induction of chondrogenic differentiation of MSCs on a glycosaminoglycan-mimetic environment produced through the supramolecular assembly of peptide amphiphile (PA) molecules that present specific functional epitopes such as sulfonate, carboxylate and hydroxyl groups in high densities across one-dimensional arrays ${ }^{26-29}$ (Figure 1, Table S1). The PA molecules contain an alkyl tail attached to peptide domain, and in aqueous environment, hydrophobic collapse of alkyl tails drive the self-assembly of PA molecules, which results in nanofiber formation. ${ }^{30-33}$ PA molecules functionalized with sulfonate, carboxylate and/or hydroxyl groups self-assemble into bioactive (GAG-PA/E-PA/K-PA and GAG-PA/K-PA) and control (E-PA/K-PA) nanofiber networks with structures similar to that of the native extracellular matrix and provide suitable platforms for the culture of MSCs. In this study, the chondrogenic differentiation of MSCs was shown to be induced in the presence of glycosaminoglycan-mimetic platforms through investigation of sulfated glycosaminoglycan deposition and cartilage-specific gene and protein expression analyses. We also showed that the extent of chondrogenic differentiation was dependent on the degree of epitope density presented on the nanofiber system.

\section{EXPERIMENTAL SECTION}

2.1. Materials. 9-Fluorenylmethoxycarbonyl (Fmoc) and tertbutoxycarbonyl (Boc) protected amino acids, $\left[4-\left[\alpha-\left(2^{\prime}, 4^{\prime}\right.\right.\right.$-dimethoxyphenyl) Fmoc-aminomethyl] enoxy] acetamidonorleucyl-MBHA resin (Rink amide MBHA resin), Fmoc-Glu $(\mathrm{OtBu})-W a n g$ resin, and 2-(1HBenzotriazol-1-yl)-1,1,3,3-tetramethyluronium hexafluorophosphate (HBTU) were purchased from NovaBiochem and ABCR. All other chemicals and materials used in this study were purchased from Invitrogen, Fisher, Merck, Alfa Aesar, Thermo-Scientific, or SigmaAldrich. Cover glasses and tissue culture plates were purchased from BD. rMSCs was purchased from Invitrogen at passage 7.

2.2. Synthesis and Characterization of Peptide Amphiphile Molecules. GAG-PA (Lauryl-VVAGEGDKS-Am) and K-PA (LaurylVVAGK-Am) were synthesized on MBHA Rink Amide resin and E-PA (Lauryl-VVAGE) was synthesized on Fmoc-Glu-(OtBu)-Wang resin. Fmoc deprotection was performed in a solution of $20 \%(\mathrm{v} / \mathrm{v})$ piperidine in DMF for $20 \mathrm{~min}$. All amino acid activation and couplings were performed in 2 equiv of amino acids in $10 \mathrm{~mL}$ of DMF, 1.95 equiv of O-Benzotriazole- $\mathrm{N}, \mathrm{N}, \mathrm{N}^{\prime}, \mathrm{N}^{\prime}$-tetramethyl-uronium-hexafluorophosphate (HBTU), and 3 equiv of $\mathrm{N}$-ethyl-diisopropylamine (DIEA) for $2 \mathrm{~h}$ for every amino acid. After every coupling reactions, unreacted amines were masked by $10 \%$ acetic anhydride. Synthesized peptide amphiphile molecules were cleaved from resin in trifluoroacetic acid (TFA) triisopropylsilane (TIS):water solution. Excess DCM and TFA were removed by rotary-evaporation. The remaining peptide amphiphile solution was triturated in diethyl ether for $16 \mathrm{~h}$ at -20 ${ }^{\circ} \mathrm{C}$. Diethyl ether was removed after centrifugation at $8000 \mathrm{rpm}$. 
Peptides were identified and purified by using a reverse phase Agilent 1200 HPLC system. The stationary phase was Zorbax ExtendC18 $21.2 \times 150 \mathrm{~mm}$ column for E-PA and GAG-PA. Elution was performed in mobile phase of a linear gradient of acetonitrile for 30 min. Purities and identities of peptide amphiphiles molecules were analyzed with an Agilent 6530-1200 Q-TOF LC-MS. A Zorbax Extend-C18 $21.2 \times 150 \mathrm{~mm}$ column was used for K-PA, and Zorbax Extend C18 column was used for GAG-PA and E-PA (Figure S1).

2.3. Preparation of Nanofibrous Networks. An aqueous solution of peptide amphiphiles was prepared in double distilled water at $\mathrm{pH}$ 7.4. Mixing of two oppositely charged PA solutions at 10 $\mathrm{mM}$ in specified volumetric ratios (Table S1) resulted in the formation of peptide hydrogels. Hydrogel morphology was assessed by using scanning electron microscopy (SEM). Briefly, peptide hydrogels were prepared on silicon wafer and incubated at room temperature for 15 min. After the formation of gels, samples were dehydrated in gradually increasing ethanol/water solutions and dried using a Tourismis Autosamdri-815B critical point drier. Dried samples were coated with $3 \mathrm{~nm} \mathrm{Au} / \mathrm{Pd}$ and visualized under high vacuum with a FEI Quanta 200 FEG SEM equipped with an ETD detector.

Secondary structures of nanofibers was assessed by circular dichroism analysis of $0.3 \mathrm{mM}$ aqueous solutions of PA molecules $(\mathrm{pH}$ 7.4) diluted from $2 \mathrm{mM}$ stock solutions. Circular dichroism spectra were acquired using J-815 Jasco spectrophotometer from 190 to $300 \mathrm{~nm}$. Spectra were obtained using a digital integration time of 4 $\mathrm{s}$, bandwidth of $1 \mathrm{~nm}$ and data pitch of $0.1 \mathrm{~nm}$. For each sample, three spectra were averaged and expressed as mean residue ellipticity and converted to the unit of degree $\mathrm{cm}^{2} \mathrm{dmol}^{-1}$.

2.4. rMSC Culture and the Preparation of Nanofibrous Networks for In Vitro Culture. Rat mesenchymal stem cells (rMSCs) (Invitrogen) were expanded in maintenance medium consisting of DMEM supplemented with 10\% (v/v) FBS (Invitrogen), $1 \%(\mathrm{v} / \mathrm{v})$ GlutaMAX (Invitrogen) and 1\% penicillin-streptomycin (Invitrogen). Chondrogenic differentiation was induced with StemPro Chondrogenesis Differentiation Kit (Invitrogen). All experiments were conducted with cells between passages 7-9. Cells were maintained at standard cell culture environment $\left(5 \% \mathrm{CO}_{2}, 37{ }^{\circ} \mathrm{C}\right)$ in humidified incubators. Cells were passaged at $80 \%$ confluency by Trypsin-EDTA (0.025\%) (Invitrogen) and reseeded at $3000 \mathrm{cells} / \mathrm{cm}^{2}$. The culture medium was replaced every $3-4$ days.

rMSCs were seeded on PA-coated surfaces or uncoated culture plates for in vitro analysis. Coating was performed by mixing oppositely charged $1 \mathrm{mM}$ PA solutions at a ratio of $150 \mu \mathrm{L} / \mathrm{cm}^{2}$ (Table S1). Coated plates were left under laminar flow hood to dry for $16 \mathrm{~h}$ and sterilized under UV irradiation for $30 \mathrm{~min}$ before cell seeding.

2.5. Cellular Viability and Proliferation Assays. Cellular viability was assessed by calcein AM (Invitrogen) and ethidium homodimer stainings. rMSCs were seeded at a density of 1250 cells/ $\mathrm{cm}^{2}$ and cultured for 24 and $48 \mathrm{~h}$ prior to staining. At the time of assay, medium was discarded and the plate was briefly centrifuged to settle dead cells. Live and dead cells were stained with calcein AM (2 $\mu \mathrm{M})$ /ethidium homodimer $(4 \mu \mathrm{M})$ cocktail in PBS for 30 min at room temperature; viable and dead cells were subsequently imaged under light microscope and counted using ImageJ software. Fifteen random images were taken for each experimental and control groups and the average number of cells on each well calculated.

Proliferating cells were detected by Click-iT EdU assay (Molecular Probes) at day 1. rMSCs were seeded on nanofibers or uncoated culture plate at a density of 1250 cells $/ \mathrm{cm}^{2}$ in maintenance medium. After $6 \mathrm{~h}$, maintenance medium was changed with medium supplemented with $10 \mathrm{mM}$ EdU. At the time of assay, cells were fixed with $4 \%$ paraformaldehyde in PBS for $15 \mathrm{~min}$ at room temperature and after removing the fixative, cells were washed and permeabilized with $5 \%$ Triton-X for $15 \mathrm{~min}$ at room temperature. Cells were treated with Alexafluor-488 conjugated azide to detect the incorporation of EdU in replicating DNA strands. Cells stained with Alexafluor-488 were imaged by fluorescence microscopy and quantified with ImageJ software. Fifteen random images were taken for each experimental and control groups and the average number of cells on each well calculated. Proliferation rates of cells on PA-coated surfaces were normalized against cells cultured on uncoated culture plate.

2.6. Glycosaminoglycan Imaging and Quantification. Glycosaminoglycan deposition was assessed through Safranin-O staining. Safranin-O is a cationic dye that binds to negatively charged sulfated glycosaminoglycans. First culture medium was removed and cells were washed with PBS. To keep morphology, cells were fixed with $4 \%$ paraformaldehyde for $15 \mathrm{~min}$ at room temperature. Then, fixed cells were blocked with $2 \%$ BSA in PBS for $30 \mathrm{~min}$. Cells were then stained with $0.1 \%(\mathrm{w} / \mathrm{v})$ Safranin-O in $0.1 \%(\mathrm{v} / \mathrm{v})$ acetic acid for $5 \mathrm{~min}$ at room temperature. To remove unbound dye, we performed extensive washing with PBS.

Quantification of sulfated glycosaminoglycans was performed using a biochemical dimethylmethylene blue (DMMB) assay. Briefly, after removing culture medium, cells were washed with PBS. Cells were digested in papain digestion buffer containing $100 \mathrm{mM}$ sodium phosphate buffer, $10 \mathrm{mM} \mathrm{Na}{ }_{2}$ EDTA, $10 \mathrm{mM}$ L-cysteine and $0.125 \mathrm{mg} /$ $\mathrm{mL}$ papain for $16 \mathrm{~h}$ at $65^{\circ} \mathrm{C}$. To measure GAG production per DNA content, we also measured total DNA per well. DNA amount was identified with Qubit dsDNA quantification kit (Invitrogen) according to manufacturer's instructions. GAG amount was calculated from a standard curve that was generated using diluted chondroitin sulfate standards (from 0 to $35 \mu \mathrm{g} \mathrm{mL}^{-1}$ ). DMMB dye was prepared from 16 $\mathrm{mg} \mathrm{L}^{-1}$ 1,9-dimethylmethylene blue, $40 \mathrm{mM}$ glycine, $40 \mathrm{mM} \mathrm{NaCl}, 9.5$ $\mathrm{mM} \mathrm{HCl}(\mathrm{pH} 3.0)$ and $100 \mu \mathrm{L}$ of dye solution was added onto $40 \mu \mathrm{L}$ of papain-digested solutions and standard samples. Then optical densities (ODs) were measured using a $595 \mathrm{~nm}$ filter on a microplate reader. The absorbance of the cell-free control groups was subtracted from the absorbance values of the experimental groups.

2.7. Gene Expression Analysis. Quantitative real time PCR (qRT-PCR) was used to analyze gene expression profiles of differentiating rMSCs. Before qRT-PCR experiments, RNA from each sample was extracted by TRIzol reagent (Invitrogen) according to manufacturer's instructions. RNA yield and purity were assessed by Nanodrop 2000 system (Thermoscientific). SuperScript III Platinum SYBR Green One-Step qRT-PCR Kit (Invitrogen) was used for cDNA synthesis and qRT-PCR that take place at the same tube. According to manufacturer's instructions reaction was set as $55^{\circ} \mathrm{C}$ for $5 \mathrm{~min}, 95^{\circ} \mathrm{C}$ for $5 \mathrm{~min}, 40$ cycles of $95{ }^{\circ} \mathrm{C}$ for $15 \mathrm{~s}, 60^{\circ} \mathrm{C}$ for $30 \mathrm{~s}$, and $40{ }^{\circ} \mathrm{C}$ for 1 min. To confirm product specificity, we performed melting curve analysis after each reaction. Before gene expression analysis, a standard curve with 5-fold serial dilutions of total RNA were generated to evaluate reaction efficiencies of each primer set. Each run was internally normalized to GAPDH, and each group was normalized to the expression levels of MSCs cultured in maintenance medium. A comparative $\mathrm{Ct}$ method with efficiency correction was used to analyze the results. Expression ratios higher than one indicate the upregulation of the gene of interest, whereas ratios less than 1 correspond to its downregulation.

2.8. Immunostaining and Imaging. rMSCs cultured on peptide coated or uncoated glass surfaces at day 7 were fixed in $4 \%$ paraformaldehyde/PBS for $10 \mathrm{~min}$ and permeabilized in $0.1 \%$ Triton X-100 for 15 min. For blocking, samples were incubated with $10 \%$ (w/ v) bovine serum albumin/PBS for $30 \mathrm{~min}$ and treated with collagen II primary antibody (Abcam) at 1:200 dilution or aggrecan antibody at 1:200 dilution (Abcam) overnight at $4{ }^{\circ} \mathrm{C}$. Cells were then washed with PBS and incubated for $1 \mathrm{~h}$ at room temperature with Goat AntiRabbit IgG H\&L (Alexa Fluor 488). All samples were counterstained with $1 \mu \mathrm{M}$ TO-PRO-3 (Invitrogen) in PBS for $15 \mathrm{~min}$ at room temperature and mounted with Prolong Gold Antifade Reagent (Invitrogen) with a coverslip. Negative controls were obtained by incubating the samples with $1 \%$ normal goat serum/PBS instead of primary antibody. Samples were imaged using Zeiss LSM510 system.

2.9. Statistical Analysis. All data are presented as means \pm standard error of means (s.e.m). One-way ANOVA and Bonferronni post-test were performed to test the significance of observed differences between the study groups. " $n$ " denotes experimental replicates and $n=3$. A $p$ value of less than 0.05 was considered to be statistically significant, except where noted. 
A.
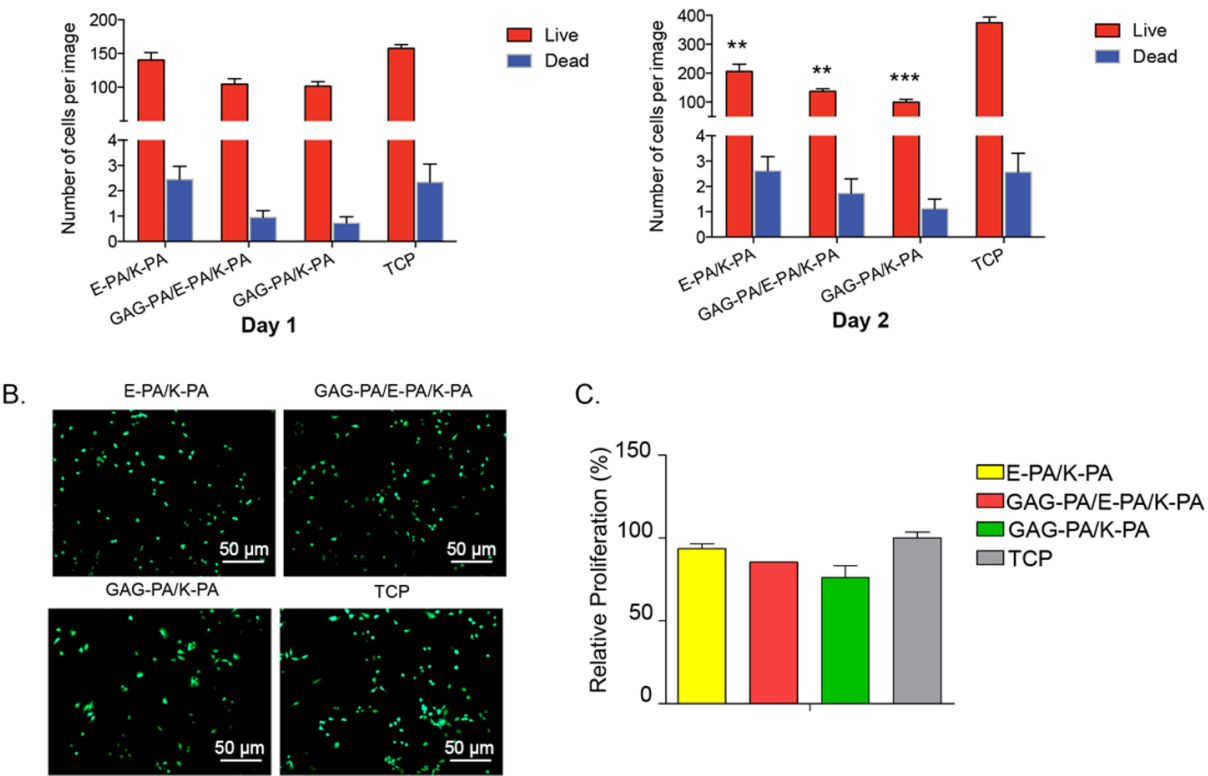

Figure 2. Viability and Proliferation of MSCs on nanofiber networks at $24 \mathrm{~h}$. (A) Viability and (C) proliferation rates of MSCs on nanofiber networks and bare culture plates. (** or *** denotes statistical analysis result between TCP and E-PA/K-PA or GAG-PA/E-PA/K-PA or GAG-PA/ K-PA.) (B) Representative Calcein-AM stained micrographs of MSCs at $24 \mathrm{~h}$.

\section{RESULTS AND DISCUSSION}

3.1. Characterization of Peptide Amphiphile Nano-

fibers. In this study, we aimed to mimic function of heparan sulfate glycosaminoglycans in the extracellular matrix by incorporating functional units found in heparan sulfate, such as carboxylate, sulfonate and hydroxyl groups, into a peptide nanofiber network. High-aspect-ratio nanofibers were produced by mixing oppositely charged peptide amphiphile molecules at molar ratios given at Table S1. The amphiphilic nature of peptide amphiphile molecules facilitated the formation of onedimensional nanofibers through the hydrophobic collapse of the alkyl tails, intermolecular hydrogen bonding of hydrophobic amino acids in the form of $\beta$-sheets and electrostatic interactions between the charged amino acids. ${ }^{28,34}$ This particular geometry of nanofibers allows the presentation of high-density functional epitopes on the outer periphery of the peptide nanofibers. Three different peptide amphiphile molecules were used to form three different nanofiber networks. Lauryl-VVAGE (E-PA) carried carboxylate groups as its functional units, while glycosaminoglycan mimetic LaurylVVAGEGD-K(p-sulfobenzoyl)-S-Am (GAG-PA) carried sulfonate, carboxylate and hydroxyl groups and Lauryl-VVAGK-Am (K-PA) was a positively charged peptide amphiphile molecule used to induce nanofiber formation with negatively charged peptide amphiphiles (Figure 1A). The GAG-PA molecule carrying sulfonate, carboxylate and hydroxyl groups was previously designed by our group and its activity in angiogenesis, and cellular differentiation was shown. ${ }^{29,35}$ Furthermore, it was shown that glycosaminoglycan mimetic peptide nanofiber networks encapsulate growth factors and increase their local concentrations. ${ }^{36}$

Nanofiber networks were formed by mixing PA molecules at different concentrations to adjust the presentation of functional groups at different ratios. The E-PA/K-PA did not contain sulfonate groups and served as a control for sulfonate functionality, while GAG-PA/K-PA and GAG-PA/E-PA/K-PA bore all the functional group types and was used as GAG- mimetic nanofiber networks; however, GAG-PA/E-PA/K-PA contained less sulfonate groups $(1 \times)$ compared to GAG-PA/KPA $(2 \times)$ (Table S1). In all of these systems, we observed dense and interconnected organization of nanofibers resulting in the formation of networks that closely resembles the nanofibrous morphology of the native extracellular matrix and there was no significant morphological difference between the nanofiber networks (Figure 1C). In culture conditions, coatings were quite stable that there were no delamination or rupture during the course of experiment.

When two oppositely charged peptide amphiphiles were mixed, the $\beta$-sheet structure was the dominant secondary structure of the resulting nanofibers, as shown by circular dichroism spectra showing a maximum around $200 \mathrm{~nm}$ and minimum around $220 \mathrm{~nm}$ (Figure $1 \mathrm{~B}$ ). The peptide amphiphile molecules E-PA and GAG-PA, showed random coil (Figure S2) and K-PA showed $\beta$-sheet structure at $\mathrm{pH} 7.4$ when they were measured alone.

3.2. Cellular Behavior on Nanofiber Network. We investigated the biocompatibility of nanofiber networks by examining the viability of MSCs cultured on peptide scaffolds for 24 and $48 \mathrm{~h}$. Calcein AM staining was performed to determine the number of viable cells and ethidium homodimer staining was performed to determine the number of dead cells. Bare tissue culture plates were used as a control. Lower numbers of cells were stained with Calcein AM for MSCs cultured on nanofiber groups at 24 and $48 \mathrm{~h}$ compared to MSCs on bare tissue culture plate surface (Figure 2A, B). In accordance with viability results, the proliferation rate of MSCs on nanofiber scaffolds was also lower compared to bare tissue culture plate (Figure 2C). The low number of proliferating cells may explain the lower number of viable cells on nanofiber scaffolds, as the decreased proliferation of MSCs would lead to a lower number of cells present on nanofiber scaffolds at 24 and $48 \mathrm{~h}$. It is known that stem cells decrease their proliferation rate under environments that are inductive for their differentiation. ${ }^{37}$ The response of MSCs cultured on nanofiber 
scaffolds may be directed toward differentiation from the onset of their seeding, which would result in a decrease in the number of proliferating cells. Few dead cells also supports that low number of viable cells may be the result of differentiation rather than toxicity. Furthermore, differences in proliferation rate at the $48 \mathrm{~h}$ time period might be the result of heterogeneity in initial MSC population and plastic properties of MSCs that contribute to phenotypic and functional variances of cultures on different surfaces. ${ }^{38}$

3.3. Peptide Nanofiber Networks Promote MSC Aggregation and Deposition of Cartilage Extracellular Matrix Components. We then analyzed the chondrogenic differentiation potential of MSCs on nanofiber networks bearing different functional epitopes. MSCs that commit to the chondrocytic lineage rapidly lose their fibroblastic morphology, deposit sulfated glycosaminoglycans and increase cell-cell interactions, as evidenced by aggregate formation. $^{39-41}$ At day 7, MSCs on nanofiber networks displayed morphological similarities to chondrocytes, acquiring a rounded morphology and forming aggregate units (Figure S3). These cell aggregates were distinct and homogeneously distributed on GAG-PA/E-PA/K-PA and E-PA/K-PA systems. In contrast to these groups, aggregates formed on GAG-PA/K-PA were smaller in size. As such, differences in cellular responses exist with respect to nanofiber composition. On bare culture plates, MSCs preserved their fibroblastic/spindle shapes over 7 days even in the presence of chondrogenic medium (Figure S3).

The deposition of glycosaminogycans was examined by Safranin-O staining and dimethylmethylene blue (DMMB) assay on day 7. Discrete staining on or around cellular aggregates was clear for MSCs on nanofiber networks and indicated the accumulation of glycosaminoglycans in both maintenance and chondrogenic medium, whereas cells on bare culture plates were stained less prominently (Figure 3A). Quantitatively, the accumulation of glycosaminoglycans was higher in cells on all three nanofiber networks compared to



B.

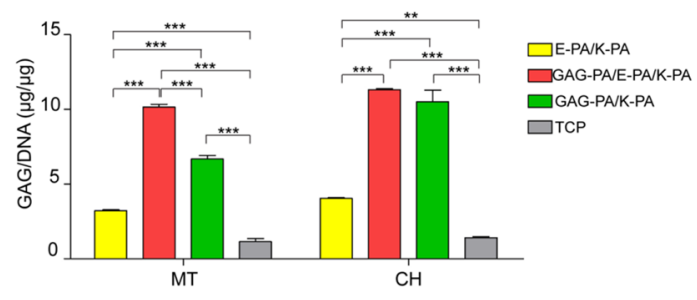

Figure 3. Glycosaminoglycan deposition of MSCs on nanofiber networks or uncoated tissue culture plates (TCP) on day 7. (A) Safranin-O staining and (B) DMMB assay on day 7 when cells were cultured in either chondrogenic $(\mathrm{CH})$ or maintenance $(\mathrm{MT})$ medium. GAG content was normalized to DNA content and expressed as $\mu \mathrm{g} /$ $\mu \mathrm{g}$. Values represent mean $\pm \mathrm{SEM}, n=3(* * * p<0.0001, * * p<0.01$, $\left.*_{p}<0.05\right)$. cells cultured on uncoated tissue culture plates. These results suggest that all nanofiber networks promoted GAG deposition by MSCs (Figure 3B). When GAG deposition of cells on different nanofiber networks were compared, we observed that GAG production in both sulfonate containing groups, GAGPA/E-PA/K-PA and GAG-PA/K-PA, were higher compared to the group that does not contain sulfonate, E-PA/K-PA. This result shows the importance of the presence of sulfonate group for the induction of GAG production. In addition, since both GAG-PA/E-PA/K-PA and GAG-PA/K-PA contain the same functional epitopes but at different presentation density (Table S1), higher GAG deposition of cells on GAG-PA/E-PA/K-PA compared to cells on GAG-PA/K-PA further reveals the importance of optimal ligand density on nanofiber surface.

3.4. Gene Expression Profiles of MSCs Confirm Chondrogenic Lineage Commitment. To elicit the importance of proper functional epitope type and amount presented on nanofibers to MSCs, we also performed Collagen II, Aggrecan, and Collagen I expression analysis of MSCs cultured on different peptide nanofiber networks that displayed different epitope densities (Table S3). Morphological observation clearly showed that MSCs formed aggregates within 3 days following their seeding on peptide nanofibers, suggesting a rapid commitment to the chondrogenic lineage. Morphological changes were confirmed by gene expression pattern analyses on both day 3 and day 7. MSCs exhibited an up-regulation of Collagen II (Day $3 \approx 4$ folds, Day $7 \approx 15$ folds) and Aggrecan (Day $3 \approx 6$ folds, Day $7 \approx 12$ folds), two predominant components of cartilage extracellular matrix, on GAG-PA/EPA/K-PA nanofiber networks at days 3 and 7 (Figure 4A, B). With regards to the reference gene, GAPDH, the relative expression of Collagen II and Aggrecan were significantly higher compared to E-PA/K-PA and GA-PA/K-PA groups. Collagen II/I ratio is another widely used differentiation index and is expected to be higher than 1 for cells committing to the chondrogenic lineage. ${ }^{42}$ The collagen phenotype of differentiated chondrocytes is marked by the predominant synthesis of Collagen II, whereas the MSC or dedifferentiated phenotype is composed primarily of Collagen I, as differentiating cells increase their Collagen II expression while decreasing Collagen I expression. In agreement with Collagen II and Aggrecan expression results, the Collagen II/I ratio was higher for MSCs cultured on GAG-PA/E-PA/K-PA at days 3 ( $\sim 7$ folds) and 7 ( $\sim 95$ folds) (Figure 4A, B). Even when cells were cultured in maintenance media (which includes no chondrogenic cues, but contains serum to encourage cell proliferation), the GAG-PA/ E-PA/K-PA group still showed an upregulated expression of Collagen II ( $\sim 7$ folds) and higher fold changes of Collagen II/I ( $\sim 32$ folds) at day 7 (Figure S4B). In parallel with gene expression patterns, MSCs on nanofibers showed enhanced protein expression compared to cells on bare culture plate (Figure 4C).

To further investigate the effect of sulfonate epitope density on chondrogenic differentiation of MSCs, MSCs were cultured on nanofiber networks displaying sulfonate epitope at higher $(\mathrm{H}-1.33 x)$ and lower (L-0.66x) stoichiometric ratios than GAG-PA/E-PA/K-PA network ( $1 x$ ) (Table S3). Both the higher and lower sulfonate ratio groups showed lower expression of chondrogenesis-related markers compared to $1 x$ GAG-PA/E-PA/K-PA network (Figure 5). This result showed that epitope density affect cellular behavior in both ways as such; MSCs decrease expression of markers on either networks presenting sulfonate groups in more stack or more dilute form. 

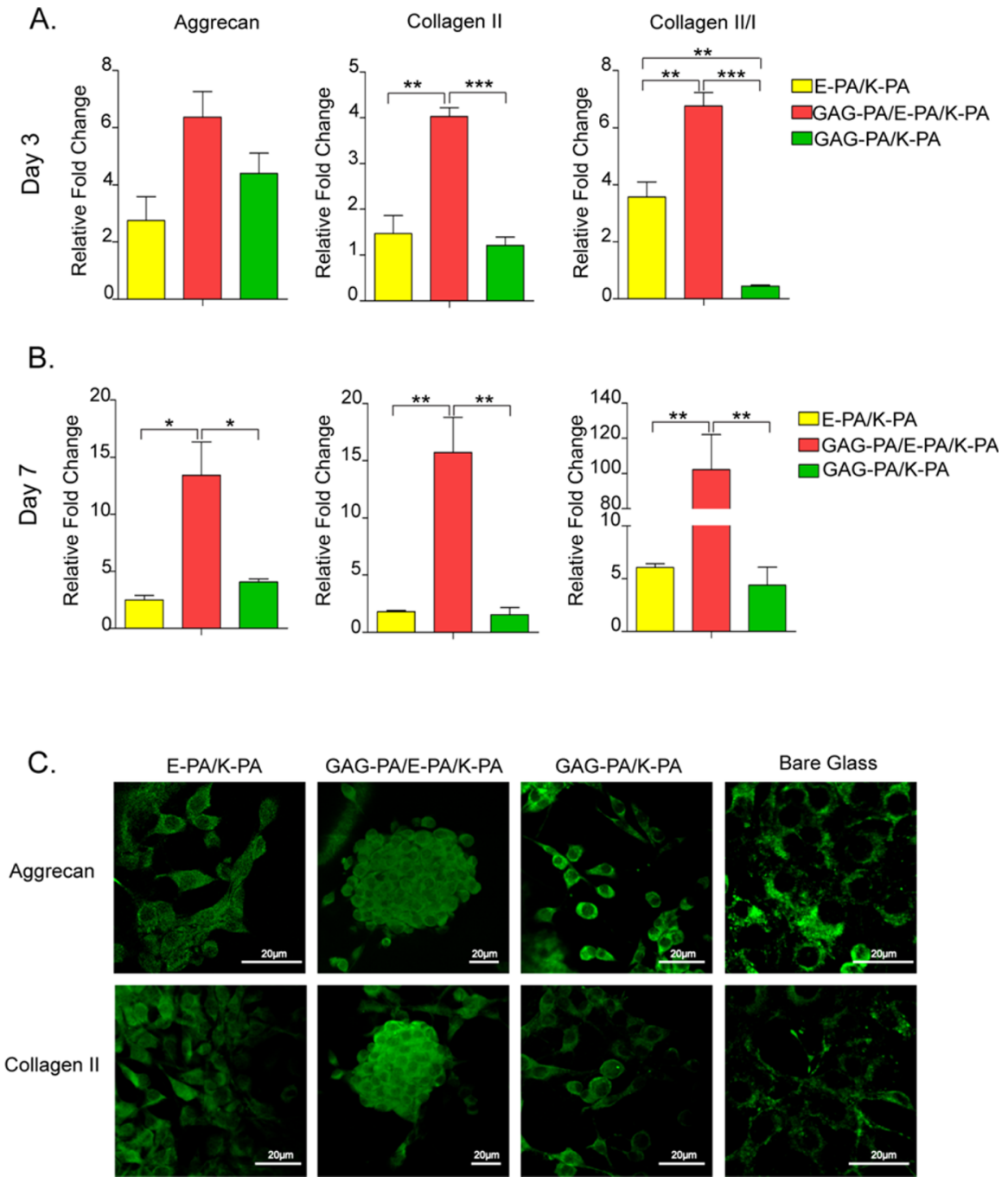

Figure 4. Cartilage specific gene and protein expression. (A, B) Aggrecan, Collagen II, and Collagen II/I expression of MSCs on nanofiber networks on day 3 and 7 in chondrogenic medium. The expression level of each gene was normalized against TCP and GAPDH was used as the internal control. Values represent mean \pm SEM, $n=3(* * * p 0.0001, * * p<0.01, * p<0.05)$. (C) Aggrecan and Collagen II protein expression of MSCs on day 7 in chondrogenic medium.

We observed that it is important for cells to access optimal amounts of bioactive epitopes in available space to evoke cellular responses as shown previously in the literature. ${ }^{43}$ These results are also in agreement with studies investigating the impact of sulfation and importance of its pattern on regulation of cellular activities. ${ }^{44,45}$ The supramolecular nanofiber system enabled us to manipulate functional group concentration for controlled cell response similar to what is observed in ECM for regulated cellular activities through receptor clustering and ligand density.

\section{CONCLUSION}

Here, we studied the effect of the synthetic GAG mimetic extracellular environment on the in vitro chondrogenic differentiation of mesenchymal stem cells. The main motivation stems from the biofunctional role of GAG molecules in the promotion of chondrogenic differentiation of stem cells. Prior reports showed that heparin itself or heparin-incorporating biomaterials were able to induce lineage commitment of stem cells through growth factor sequestration and presentation to cells. ${ }^{46,47}$ Incorporating functional moieties of GAG molecules into a biomaterial may replicate such functions through the localization of endogeneous growth factors via charge interactions, which in turn offers a route to locally amplify biomolecular signals for differentiation.

We investigated three different peptide nanofiber networks, each of which mimicked the structural and fibrous characteristics of the extracellular matrix. The design of PA networks simply relied on incorporation of charged groups of native glycosaminoglycan molecules onto PA molecules that enhances localization of endogenously released positively charged biologically active molecules. ${ }^{36}$ To examine the influence of each functional group found in GAG molecules in detail, we used networks that bear carboxylate, sulfonate and hydroxyl groups at different stoichiometric ratios. E-PA/K-PA, which contains carboxylate groups, served as a negative control for sulfonate groups and GAG-PA/K-PA, a peptide scaffold that contains both sulfonate and carboxylate groups at higher stoichiometric ratios compared to GAG-PA/E-PA/K-PA, was used to investigate the effect of ligand density. Results showed that the superior chondrogenic potential of GAG-PA/E-PA/KPA was primarily attributed to the synergistic effect of carboxylate, sulfonate and hydroxyl groups in one system at proper density. This result is in accordance with our previous 

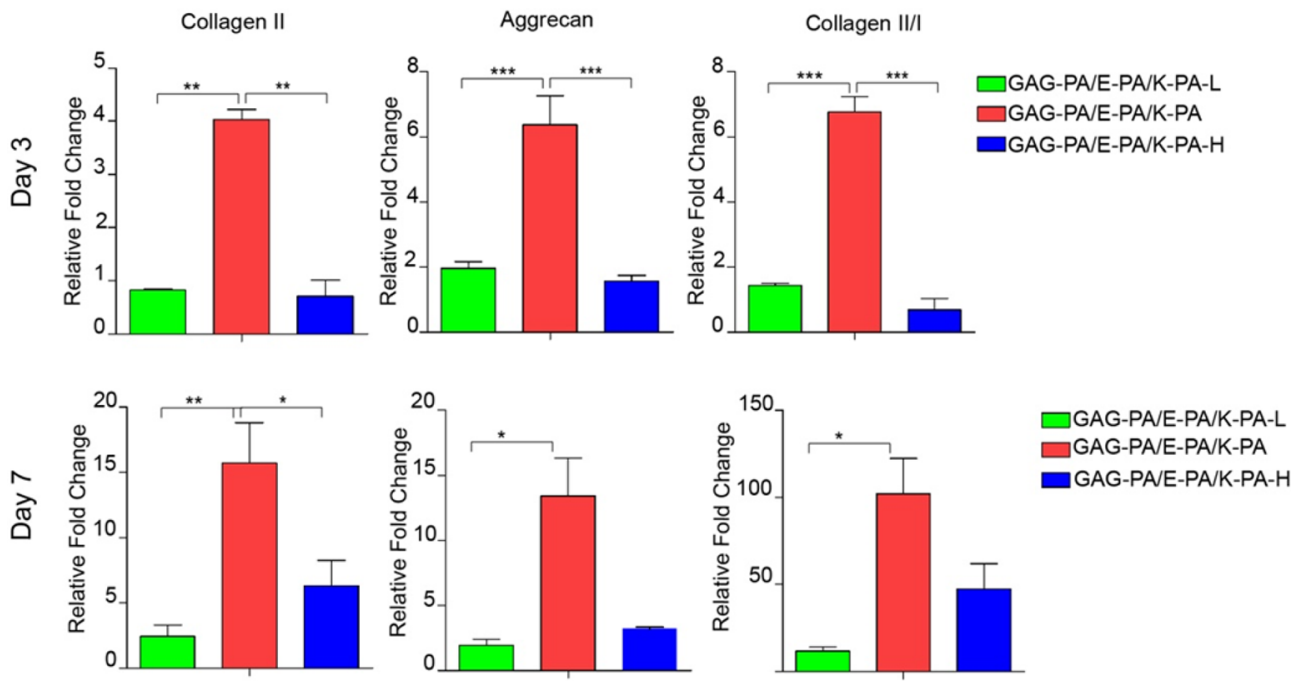

Figure 5. Cartilage specific gene expression on bioactive nanofibers with different epitope concentrations. Aggrecan, Collagen II, and Collagen II/I expression of rMSCs on nanofiber networks (GAG-PA/E-PA/K-PA-L, GAG-PA/E-PA/K-PA, and GAG-PA/E-PA/K-PA-H) on day 3 and 7 in chondrogenic medium. $\mathrm{H}$ stands for higher stoichiometric ratio of sulfonate group, $\mathrm{L}$ stands for lower stoichiometric ratio of sulfonate group compared to GAG-PA/E-PA/K-PA (Table S3). The expression level of each gene was normalized against TCP and GAPDH was used as the internal control. Values represent mean \pm SEM, $n=3(* * * p<0.0001, * * p<0.01, * p<0.05)$.

study with ATDC5 cells, which suggested that the synergistic effect of sulfonate, carboxylate, and hydroxyl groups was more effective for inducing chondrogenic differentiation using peptide nanofibers. ${ }^{48}$ Future studies may consider more specific GAG-mimetic PA molecule designs to bring improved specificity over growth factor sequestration and release.

\section{ASSOCIATED CONTENT}

\section{S Supporting Information}

The Supporting Information is available free of charge on the ACS Publications website at DOI: 10.1021/acsbiomaterials.6b00099.

$$
\text { Additional experimental files (PDF) }
$$

\section{AUTHOR INFORMATION}

\section{Corresponding Authors}

*E-mail: moguler@unam.bilkent.edu.tr.

*E-mail: atekinay@unam.bilkent.edu.tr.

\section{Notes}

The authors declare no competing financial interest.

\section{ACKNOWLEDGMENTS}

This work was supported by TUBITAK grant 111M710. A.B.T. and M.O.G. acknowledge support from the Turkish Academy of Sciences Distinguished Young Scientist Award (TUBAGEBIP). S.U.Y. is supported by TUBITAK BIDEB $\mathrm{PhD}$ fellowship.

\section{REFERENCES}

(1) Barry, F. P. Biology and clinical applications of mesenchymal stem cells. Birth Defects Res., Part C 2003, 69 (3), 250-256.

(2) Barry, F. P.; Murphy, J. M. Mesenchymal stem cells: clinical applications and biological characterization. Int. J. Biochem. Cell Biol. 2004, 36 (4), 568-584.

(3) Diao, H.; Wang, J.; Shen, C.; Xia, S.; Guo, T.; Dong, L.; Zhang, C.; Chen, J.; Zhao, J.; Zhang, J. Improved cartilage regeneration utilizing mesenchymal stem cells in TGF-betal gene-activated scaffolds. Tissue Eng., Part A 2009, 15 (9), 2687-2698.
(4) Manning, C. N.; Schwartz, A. G.; Liu, W.; Xie, J.; Havlioglu, N.; Sakiyama-Elbert, S. E.; Silva, M. J.; Xia, Y.; Gelberman, R. H.; Thomopoulos, S. Controlled delivery of mesenchymal stem cells and growth factors using a nanofiber scaffold for tendon repair. Acta Biomater. 2013, 9 (6), 6905-6914.

(5) Mohan, N.; Nair, P. D.; Tabata, Y. Growth factor-mediated effects on chondrogenic differentiation of mesenchymal stem cells in 3D semi-IPN poly(vinyl alcohol)-poly(caprolactone) scaffolds. J. Biomed. Mater. Res., Part A 2010, 94 (1), 146-159.

(6) Sundelacruz, S.; Kaplan, D. L. Stem cell- and scaffold-based tissue engineering approaches to osteochondral regenerative medicine. Semin. Cell Dev. Biol. 2009, 20 (6), 646-655.

(7) Danišovič, L.; Varga, I.; Polák, S. Growth factors and chondrogenic differentiation of mesenchymal stem cells. Tissue Cell 2012, 44 (2), 69-73.

(8) Alberti, K.; Davey, R. E.; Onishi, K.; George, S.; Salchert, K.; Seib, F. P.; Bornhäuser, M.; Pompe, T.; Nagy, A.; Werner, C.; et al. Functional immobilization of signaling proteins enables control of stem cell fate. Nat. Methods 2008, 5 (7), 645-650.

(9) Mann, B. K.; Schmedlen, R. H.; West, J. L. Tethered-TGF-beta increases extracellular matrix production of vascular smooth muscle cells. Biomaterials 2001, 22 (5), 439-444.

(10) Richardson, T. P.; Peters, M. C.; Ennett, A. B.; Mooney, D. J. Polymeric system for dual growth factor delivery. Nat. Biotechnol. 2001, 19 (11), 1029-1034.

(11) Sohier, J.; Vlugt, T. J. H.; Cabrol, N.; Van Blitterswijk, C.; de Groot, K.; Bezemer, J. M. Dual release of proteins from porous polymeric scaffolds. J. Controlled Release 2006, 111 (1-2), 95-106.

(12) Bowen-Pope, D. F.; Malpass, T. W.; Foster, D. M.; Ross, R. Platelet-derived growth factor in vivo: levels, activity, and rate of clearance. Blood 1984, 64 (2), 458-469.

(13) Naldini, L.; Vigna, E.; Narsimhan, R. P.; Gaudino, G.; Zarnegar, R.; Michalopoulos, G. K.; Comoglio, P. M. Hepatocyte growth factor (HGF) stimulates the tyrosine kinase activity of the receptor encoded by the proto-oncogene c-MET. Oncogene 1991, 6 (4), 501-504.

(14) Poynton, A. R.; Lane, J. M. Safety profile for the clinical use of bone morphogenetic proteins in the spine. Spine (Philadelphia) 2002, 27 (16 Suppl 1), S40-S48.

(15) Lee, K.; Silva, E. A.; Mooney, D. J. Growth factor delivery-based tissue engineering: general approaches and a review of recent developments. J. R. Soc., Interface 2011, 8 (55), 153-170.

(16) Park, H.; Temenoff, J. S.; Holland, T. A.; Tabata, Y.; Mikos, A. G. Delivery of TGF-betal and chondrocytes via injectable, 
biodegradable hydrogels for cartilage tissue engineering applications. Biomaterials 2005, 26 (34), 7095-7103.

(17) Ayerst, B. I.; Day, A. J.; Nurcombe, V.; Cool, S. M.; Merry, C. L. R. New strategies for cartilage regeneration exploiting selected glycosaminoglycans to enhance cell fate determination. Biochem. Soc. Trans. 2014, 42 (3), 703-709.

(18) Chen, W.-C.; Yao, C.-L.; Chu, I.-M.; Wei, Y.-H. Compare the effects of chondrogenesis by culture of human mesenchymal stem cells with various type of the chondroitin sulfate C. J. Biosci. Bioeng. 2011, 111 (2), 226-231.

(19) Goude, M. C.; McDevitt, T. C.; Temenoff, J. S. Chondroitin sulfate microparticles modulate transforming growth factor- $\beta 1$-induced chondrogenesis of human mesenchymal stem cell spheroids. Cells Tissues Organs 2014, 199 (2-3), 117-130.

(20) Guo, Y.; Yuan, T.; Xiao, Z.; Tang, P.; Xiao, Y.; Fan, Y.; Zhang, $\mathrm{X}$. Hydrogels of collagen/chondroitin sulfate/hyaluronan interpenetrating polymer network for cartilage tissue engineering. J. Mater. Sci.: Mater. Med. 2012, 23 (9), 2267-2279.

(21) French, M. M.; Gomes, R. R.; Timpl, R.; Höök, M.; Czymmek, K.; Farach-Carson, M. C.; Carson, D. D. Chondrogenic activity of the heparan sulfate proteoglycan perlecan maps to the $\mathrm{N}$-terminal domain I. J. Bone Miner. Res. 2002, 17, 48-55.

(22) DeAngelis, P. L. Glycosaminoglycan polysaccharide biosynthesis and production: today and tomorrow. Appl. Microbiol. Biotechnol. 2012, 94 (2), 295-305.

(23) Ikeda, Y.; Charef, S.; Ouidja, M.-O.; Barbier-Chassefière, V.; Sineriz, F.; Duchesnay, A.; Narasimprakash, H.; Martelly, I.; Kern, P.; Barritault, D.; et al. Synthesis and biological activities of a library of glycosaminoglycans mimetic oligosaccharides. Biomaterials 2011, 32 (3), 769-776.

(24) Da Costa, D. S.; Pires, R. a.; Frias, A. M.; Reis, R. L.; Pashkuleva, I. Sulfonic groups induce formation of filopodia in mesenchymal stem cells. J. Mater. Chem. 2012, 22 (15), 7172.

(25) Kwon, H. J.; Yasuda, K. Chondrogenesis on sulfonate-coated hydrogels is regulated by their mechanical properties. J. Mech. Behav. Biomed. Mater. 2013, 17, 337-346.

(26) Cui, H.; Webber, M. J.; Stupp, S. I. Self-assembly of peptide amphiphiles: From molecules to nanostructures to biomaterials. Biopolymers 2010, 94 (1), 1-18.

(27) Silva, G. a; Czeisler, C.; Niece, K. L.; Beniash, E.; Harrington, D. a; Kessler, J. a; Stupp, S. I. Selective differentiation of neural progenitor cells by high-epitope density nanofibers. Science 2004, 303 (5662), 1352-1355.

(28) Mammadov, R.; Mammadov, B.; Toksoz, S.; Aydin, B.; Yagci, R.; Tekinay, A. B.; Guler, M. O. Heparin mimetic peptide nanofibers promote angiogenesis. Biomacromolecules 2011, 12 (10), 3508-3519.

(29) Mammadov, B.; Mammadov, R.; Guler, M. O.; Tekinay, A. B. Cooperative effect of heparan sulfate and laminin mimetic peptide nanofibers on the promotion of neurite outgrowth. Acta Biomater. 2012, 8 (6), 2077-2086.

(30) Paramonov, S. E.; Jun, H.; Hartgerink, J. D. Self-Assembly of Peptide - Amphiphile Nanofibers: The Roles of Hydrogen Bonding and Amphiphilic Packing. J. Am. Chem. Soc. 2006, 128, 7291-7298.

(31) Hartgerink, J. D.; Beniash, E.; Stupp, S. I. Self-assembly and mineralization of peptide-amphiphile nanofibers. Science 2001, 294 (5547), 1684-1688.

(32) Toksoz, S.; Acar, H.; Guler, M. O. Self-assembled onedimensional soft nanostructures. Soft Matter 2010, 6 (23), 5839.

(33) Toksöz, S.; Guler, M. O. Self-assembled peptidic nanostructures. Nano Today 2009, 4 (6), 458-469.

(34) Niece, K. L.; Hartgerink, J. D.; Donners, J. J. J. M.; Stupp, S. I. Self-assembly combining two bioactive peptide-amphiphile molecules into nanofibers by electrostatic attraction. J. Am. Chem. Soc. 2003, 125 (24), 7146-7147.

(35) Mammadov, R.; Mammadov, B.; Guler, M. O.; Tekinay, A. B. Growth factor binding on heparin mimetic peptide nanofibers. Biomacromolecules 2012, 13, 3311.
(36) Kocabey, S.; Ceylan, H.; Tekinay, A. B.; Guler, M. O. Glycosaminoglycan mimetic peptide nanofibers promote mineralization by osteogenic cells. Acta Biomater. 2013, 9 (11), 9075-9085.

(37) Cooper, G. M. Cell Proliferation in Development and Differentiation; Sinauer Associates: Sunderland, MA, 2000.

(38) Pevsner-Fischer, M.; Levin, S.; Zipori, D. The origins of mesenchymal stromal cell heterogeneity. Stem Cell Rev. 2011, 7 (3), $560-568$.

(39) Hall, B. K.; Miyake, T. Divide, accumulate, differentiate: cell condensation in skeletal development revisited. Int. J. Dev. Biol. 1995, 39 (6), 881-893.

(40) Chen, W.-C.; Wei, Y.-H.; Chu, I.-M.; Yao, C.-L. Effect of chondroitin sulphate $\mathrm{C}$ on the in vitro and in vivo chondrogenesis of mesenchymal stem cells in crosslinked type II collagen scaffolds. J. Tissue Eng. Regener. Med. 2013, 7 (8), 665-672.

(41) Goldring, M. B.; Tsuchimochi, K.; Ijiri, K. The control of chondrogenesis. J. Cell. Biochem. 2006, 97 (1), 33-44.

(42) Lee, J. W.; Kim, Y. H.; Kim, S.-H.; Han, S. H.; Hahn, S. B. Chondrogenic differentiation of mesenchymal stem cells and its clinical applications. Yonsei Med. J. 2004, 45 (Suppl), 41-47.

(43) Storrie, H.; Guler, M. O.; Abu-Amara, S. N.; Volberg, T.; Rao, M.; Geiger, B.; Stupp, S. I. Supramolecular crafting of cell adhesion. Biomaterials 2007, 28 (31), 4608-4618.

(44) Hortensius, R. A.; Harley, B. A. C. The use of bioinspired alterations in the glycosaminoglycan content of collagen-GAG scaffolds to regulate cell activity. Biomaterials 2013, 34 (31), 76457652.

(45) Lim, J. J.; Hammoudi, T. M.; Bratt-Leal, A. M.; Hamilton, S. K.; Kepple, K. L.; Bloodworth, N. C.; McDevitt, T. C.; Temenoff, J. S. Development of nano- and microscale chondroitin sulfate particles for controlled growth factor delivery. Acta Biomater. 2011, 7 (3), 986995.

(46) Benoit, D. S. W.; Anseth, K. S. Heparin functionalized PEG gels that modulate protein adsorption for hMSC adhesion and differentiation. Acta Biomater. 2005, 1 (4), 461-470.

(47) Benoit, D. S. W.; Durney, A. R.; Anseth, K. S. The effect of heparin-functionalized PEG hydrogels on three-dimensional human mesenchymal stem cell osteogenic differentiation. Biomaterials 2007, 28 (1), 66-77.

(48) Ustun, S.; Tombuloglu, A.; Kilinc, M.; Guler, M. O.; Tekinay, A. B. Growth and differentiation of prechondrogenic cells on bioactive self-assembled peptide nanofibers. Biomacromolecules 2013, 14 (1), $17-26$. 